\title{
Review
}

\section{Circadian rhythms in thrombosis and atherothrombotic events}

\author{
Eduardo Peñaloza-Martínez ${ }^{1,2}$, Guillermo Moreno ${ }^{1,3}$, Alejandra Aroca-Crevillén ${ }^{4}$, \\ Sergio Huertas ${ }^{1}$, Lourdes Vicent ${ }^{1,2}$, Nicolás Rosillo ${ }^{5}$, Andrés Hidalgo ${ }^{4}$, Héctor Bueno $^{1,2,4,6, *}$ \\ ${ }^{1}$ Cardiology Department, Hospital Universitario 12 de Octubre, and Instituto de Investigación Sanitaria Hospital 12 de Octubre (imas12), 28041 \\ Madrid, Spain \\ ${ }^{2}$ CIBERCV, Instituto de Salud Carlos III, 28029 Madrid, Spain \\ ${ }^{3}$ Facultad de Enfermería, Fisioterapia y Podología, Universidad Complutense de Madrid, 28040 Madrid, Spain \\ ${ }^{4}$ Department of Cell and Developmental Biology, Centro Nacional de Investigaciones Cardiovasculares (CNIC), 28029 Madrid, Spain \\ ${ }^{5}$ Department of Preventive Medicine. Hospital Universitario 12 de Octubre and Instituto de Investigación Sanitaria Hospital 12 de Octubre (imas12), \\ 28041 Madrid, Spain \\ ${ }^{6}$ Facultad de Medicina, Universidad Complutense de Madrid, 28040 Madrid, Spain \\ *Correspondence: hector.bueno@cnic.es (Héctor Bueno) \\ Academic Editor: Graham Pawelec \\ Submitted: 16 December 2021 Revised: 11 January 2022 Accepted: 12 January 2022 Published: 11 February 2022
}

\begin{abstract}
Biological circadian rhythms in living organisms are regulated by molecular clocks. Several of these clocks are present in blood vessels, peripheral tissues, and immune cells. There is strong evidence linking dysregulation of circadian rhythms to the development of cardiovascular disease. Dysregulation of circadian rhythms is believed to activate inflammatory processes at specific times of day, leading to an increased risk of thrombosis and atherosclerosis progression. Research into circadian clock genes and molecular networks has the potential to identify therapeutic targets to reduce cardiovascular risk. In this review, we summarize the evidence linking circadian rhythms to thrombosis and atherothrombotic events and discuss potential therapeutic implications.
\end{abstract}

Keywords: circadian clock; autonomous; shift workers; thrombosis; atherosclerosis; acute coronary syndrome; stroke

\section{Introduction}

The word circadian (from the Latin circa diem, meaning approximately a day) describes endogenous biorhythms with a periodicity of 24 hours created by the rotation of the Earth [1]. The light-dark cycle regulates the self-sustained central circadian clock in the hypothalamic suprachiasmatic nucleus ( $\mathrm{SCN}$ ), and the central clock then resets peripheral clocks in other tissues. This clock network generates biorhythms in physiological functions and behaviors [2]. A negative feedback loop allows the circadian clock to selfregulate. Organisms possessing circadian clocks can synchronize physiological and behavioral processes to cyclic environmental factors, called Zeitgebers (from the German for time giver), showing that they have extrinsic adaptive value; moreover, circadian clocks can also coordinate with internal metabolic processes, suggesting that they have intrinsic adaptive value [3].

Circadian rhythms are maintained by inputs from a number of structures. A prominent example is the retina, which captures photons and transmits information through neurons to the SCN. Once incoming stimuli are integrated by the central clock, hormonal and neuronal stimuli regulate circadian gene expression in peripheral tissues [4]. Peripheral tissues are not passive in this process; non-SCN cells in peripheral tissues are equipped with their own endogenous circadian oscillators that direct rhythmic expression of clock genes. These clock genes exert a broad-ranging con- trol over many biological processes, including fundamental metabolic pathways, such as glucose homeostasis and lipogenesis, or macrophage activity in the kidneys, liver, adipose tissue, and the vascular system [2,5].

Changes during the day are observed in a host of physiological parameters, including cardiac contractility; blood pressure; body temperature; heart rate; and electrophysiological parameters such as PR, QRS, and QTc-interval [68]. Diurnal increases in blood pressure occur twice per day, and the evening rise is mediated by the endogenous circadian clock [9]. Initially, the main regulator of circadian variations in physiological parameters was thought to be the autonomic nervous system. However, knock-out studies of clock genes in animal models and solid organ transplantation studies in humans have shown that while blockade of the autonomous nervous system diminishes rhythmicity, alterations to the molecular circadian clock induces a more general disruption of the 24-hour variation of physiological parameters. Thus, after autonomic denervation (occurring as a result of solid organ transplantation), 24-hour rhythmicity is maintained for some physiological parameters, such as heart rate, whereas whole-body genetic disruption of the molecular circadian clock blunts the diurnal variation of multiple physiological parameters [10,11].

There is evidence linking altered daily variations in these parameters to thromboembolic and cardiovascular events $[12,13]$. Thus, the disruption of circadian 
rhythms seen in shift workers and sleep-disorder patients is associated with higher cardiovascular risk and adverse health outcomes [14]. Potential mechanisms include the dysregulation of circadian rhythms that regulate immune function, including those affecting lipid metabolism, proinflammatory cytokines, and immune-cell dynamics; these changes can lead to endothelial dysfunction, dyslipidemia, increased blood vessel stiffening, and loss of the nocturnal dip in blood pressure, resulting in hypertension and atherosclerosis-mediated coronary artery or cerebrovascular disease $[15,16]$. Understanding the dysregulation of circadian rhythms and how this leads to atherothrombotic events is crucial for the development of new treatments. In this review, we highlight evidence supporting a role for circadian rhythms in thrombosis and atherothrombotic events and outline potential therapeutic implications.

\section{Atherosclerosis, thrombosis, and circadian rhythms}

Atherosclerosis is a common mechanism underlying several manifestations of cardiovascular disease, including myocardial infarction, ischemic stroke, and cardiac arrest [17]. Endothelial dysfunction and high blood cholesterol induce the expression of membrane molecules that recruit leukocytes such as monocytes and other inflammatory cells. Once activated and adhered to the endothelium, monocytes differentiate into macrophages that drive chronic inflammation, proliferation, and apoptosis and lead to the formation of an atherosclerotic plaque [18].

The molecular clock regulates diurnal production of cytokines and oscillations in the numbers of leukocyte populations, including neutrophils, short-lived cells which show an optimal adaptation to the circadian rhythms of mammals [19,20]. Rhythmic recruitment of neutrophils may also be responsible for the circadian manifestation of several inflammatory diseases [19]. Studies in myocardial ischemia mouse models have established that neutrophil infiltration increases at night (zeitgeber time [ZT] 13, or 1 hour after lights off) through a process mediated by the chemokine receptor CXCR2, accounting for the more severe cardiac damage resulting from myocardial infarction at this time [21].

Plaque rupture can produce thrombosis, in which a blood clot forms and occludes the blood vessel. Oscillating circadian patterns have been detected for some of the hemostasis molecules involved in thrombosis, including procoagulation factors such as von Willebrand factor; factors VII, VIII, IX, and X; and anticoagulation factors such as antithrombin (AT), protein $\mathrm{C}$, and protein S. Fibrinolysis molecules (D-dimers and factor X) [22] and the activity of plasminogen activator inhibitor-1 (PAI-1) show morning peaks [23]. Platelet production and aggregation is regulated by the central clock (via thrombopoietin) [24,25], with morning peaks in numbers and afternoon peaks in the activity and expression of activation molecules such as platelet factor 4 (CXCL4) and B-thromboglobulin (B-TG) [26].

Under physiological conditions, nitric oxide (NO) synthesis decreases during daylight hours, with a consequent reduction in vasodilation, and an opposite mechanism operates during the night [27]. Endothelial dysfunction linked to atherosclerosis is associated with further daytime reductions in NO synthesis, resulting in increased vasoconstriction and hypercoagulability, likely contributing to the higher incidence of cardiovascular events such as acute myocardial infarction (AMI) and stroke during morning hours [28-31]. The amplitude of the diurnal variation in blood pressure is increased in patients with hypertension, and the oscillation coincides with the temporal variability in their incidence of acute vascular events, such as AMI, sudden cardiac death, and stroke $[32,33]$. The independent effect of chronic circadian misalignment on cardiovascular disease risk factors still needs to be studied, but mounting evidence suggests that environmental factors that disrupt physiological rhythms might contribute to cardiovascular events, as well as increasing other risk factors typically associated with cardiovascular disease [34,35]. An imbalance in the circadian patterns of these molecules, inflammatory cells, the endothelium, and platelet function increases the probability of thrombosis and atherothrombotic events (Fig. 1).

\section{Circadian rhythm: clock components and vascular involvement}

The circadian clock is a cell-autonomous molecular mechanism through which clock proteins regulate circadian rhythms. The main clock proteins include the transcription factors brain-muscle aryl hydrocarbon receptor nuclear translocator-like protein 1 (BMAL1 or ARNTL) and circadian locomotor output cycles kaput (CLOCK) and the transcriptional modulators period 1/2/3 (PER1/2/3) and cryptochrome 1/2 (CRY1/2) (Fig. 2, Ref. [2,33,34,36-53]).

BMAL1 expression follows a diurnal pattern, but the phase and amplitude vary depending on the tissue and cell type. In vascular tissues such as in vascular smooth muscle cells and the mouse aorta coincides with the transition from the dark to the light phase [36], a very similar expression pattern occurs in liver [54]. In the mouse SCN and in the lateral part of the central nucleus of the amygdala, BMAL1 expression peaks at around ZT18, and in contrast, the dentate gyrus of the hippocampus BMAL1 expression peaks at around ZT2.5 (9 hours later) [55]. BMAL1 forms a heterodimer complex with CLOCK that induces the transcription of the circadian proteins PER1/2/3 and CRY1/2, especially during the daylight phase [54]. The BMAL1-CLOCK heterodimer induces transcription by binding E-box sequences in the PER $1 / 2 / 3$ and CRY $1 / 2$ promoter regions [56]. During the evening, PER $1 / 2$ and CRY $1 / 2$ associate and translocate to the nucleus [37,54]. During the night, the PER and CRY proteins are progressively phosphorylated, and the phosphorylated proteins are ubiquitinated by specific E3 ligases and eventually degraded by the proteasome 


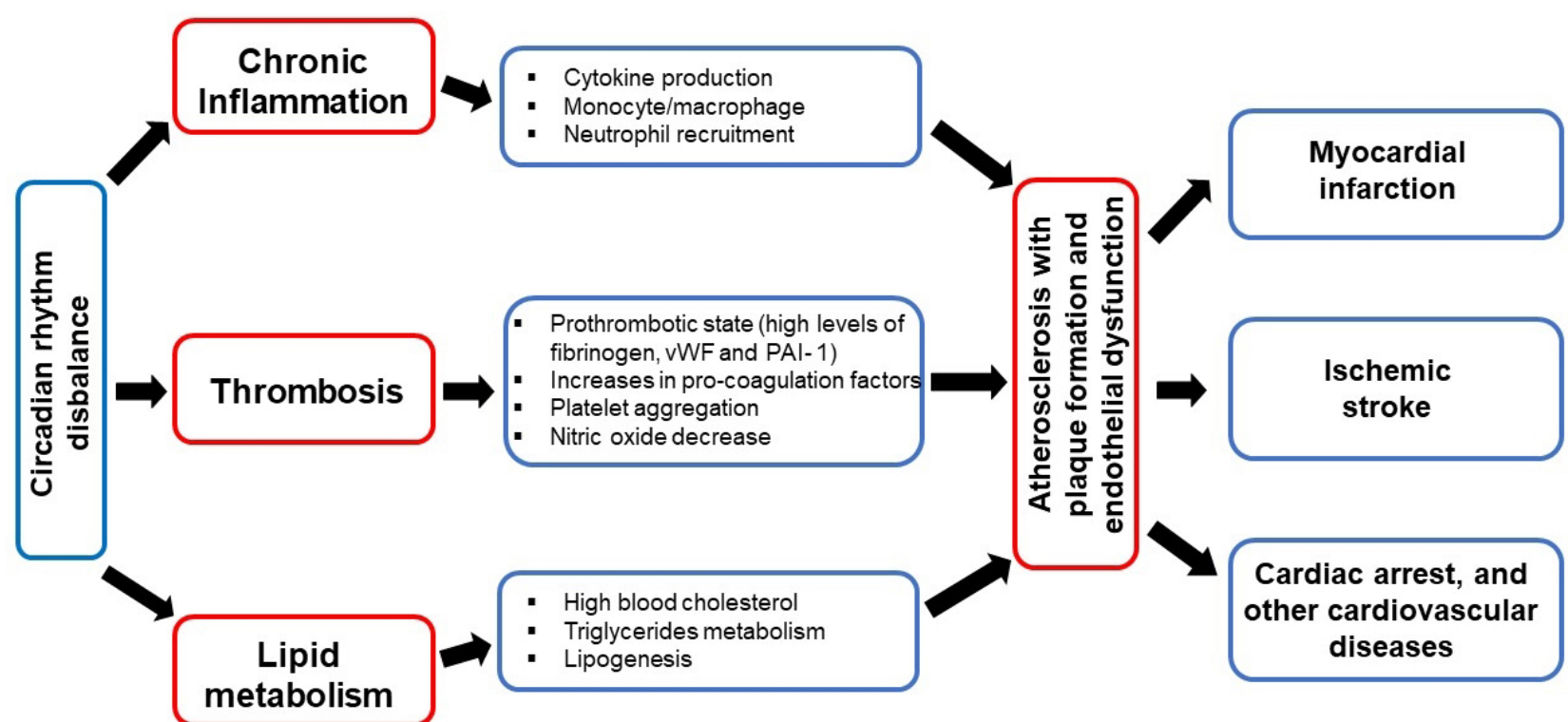

Fig. 1. Atherosclerosis, thrombosis, and circadian rhythms. Atherosclerosis is a common pathophysiological mechanism underlying cardiovascular disease. Under physiological conditions, the molecular clock regulates the diurnal production of cytokines and the recruitment of leukocytes and other inflammatory cells. The molecular clock also regulates critical lipid homeostasis mechanisms controlling cholesterol and triglyceride metabolism. Hemostasis molecules involved in thrombosis and that show a circadian rhythm include pro-coagulation factors, platelet aggregation factors, and vasorelaxation molecules. Disruption of the circadian patterns governing these molecules or controlling inflammatory cells and platelet function may contribute to endothelial dysfunction, leading to increases in thrombosis and atherothrombotic events, including myocardial infarction, ischemic stroke, and cardiac arrest.

[38]. PER $1 / 2$ and CRY1/2 repress their own transcription via a negative feedback loop after physically binding to heterodimers formed by BMAL1 with CLOCK or its paralog NPAS2 [54,57]. BMAL1 and CLOCK are basic helix-loophelix/Per-ARNT-SIM (bHLH-PAS) proteins [58], and their transcription rate and rhythmicity are subsequently regulated by feedback loops involving two classes of nuclear receptor: the retinoic acid receptor-related orphan receptors $(\operatorname{ROR} \alpha / \beta / \gamma)$, which positively regulate the expression of BMAL1 and CLOCK, and reverse ERB (REV-ERB $\alpha / \beta$ ), which negatively regulates their expression [2]. BMAL1CLOCK heterodimers also control the expression of other loop components, such as proline and acidic amino acidrich basic leucine zipper (PAR-bZIP), thyrotroph embryonic factor (TEF), D-box binding protein (DBP), and hepatic leukemia factor (HLF) [39]. The expression of genes containing D-box-cis elements, such as DBP, is controlled by the repressor E4BP4 and the PAR-bZIP transcription factors [39]. Only $22 \%$ of cycling mRNA transcripts in mammals are driven by de novo transcription, suggesting that both transcriptional and post-transcriptional mechanisms contribute to the circadian regulation of cycling mRNA levels in mammals [54]. The circadian clock regulates the transcription of around $10-15 \%$ of the genes expressed in the heart and cardiovascular system [40].

Mice lacking BMAL1-CLOCK, PER1/2/3, or CRY1/2 are prone to atherosclerosis, and several mechanisms contribute to this increased susceptibility. One involves the circadian regulation of the expression of chemokines and cytokines such as CCL2, monocyte chemoattractant protein-1 (MCP-1), IL-1, IL-6, and tumor necrosis factor- $\alpha$ (TNF- $\alpha$ ) [41,42]. TNF- $\alpha$-induced activation of nuclear factor kappa-light-chain-enhancer of activated B cells (NF- $\kappa \mathrm{B}$ ) is involved in monocytemacrophage (type Ly6chi) activation and migration to the atherosclerotic plaque in BMAL1-deficient animals [59,60]. Macrophages eliminate the exacerbated endotoxin-induced cytokine response observed at night. This activity is altered when the expression of REV-ERB $\alpha$ is compromised [19,61].

Liver metabolism, including lipid and glucose metabolic pathways and especially cholesterol and triglyceride metabolism, loses its circadian variation when BMAL1, CLOCK, or REV-ERB $\alpha / \beta$ are absent $[43,44]$. This can also occur upon PER2 loss of function, since PER2 controls peroxisome proliferating activated receptor (PPAR- $\gamma$ ), a critical regulator of lipid homeostasis [45]. In mice with diabetes, liver-specific overexpression of Cryl can lower blood glucose and increase insulin sensitivity, whereas Cry1/2-deficient mice become obese more rapidly on a high-fat diet than control mice, showing upregulation of adipocyte genes associated with lipogenesis and lipid metabolism [46]. Cry1/2- and REV-ERB $\alpha / \beta$-deficient mice have higher than normal blood glucose and impaired glucose metabolism [47,62]. 


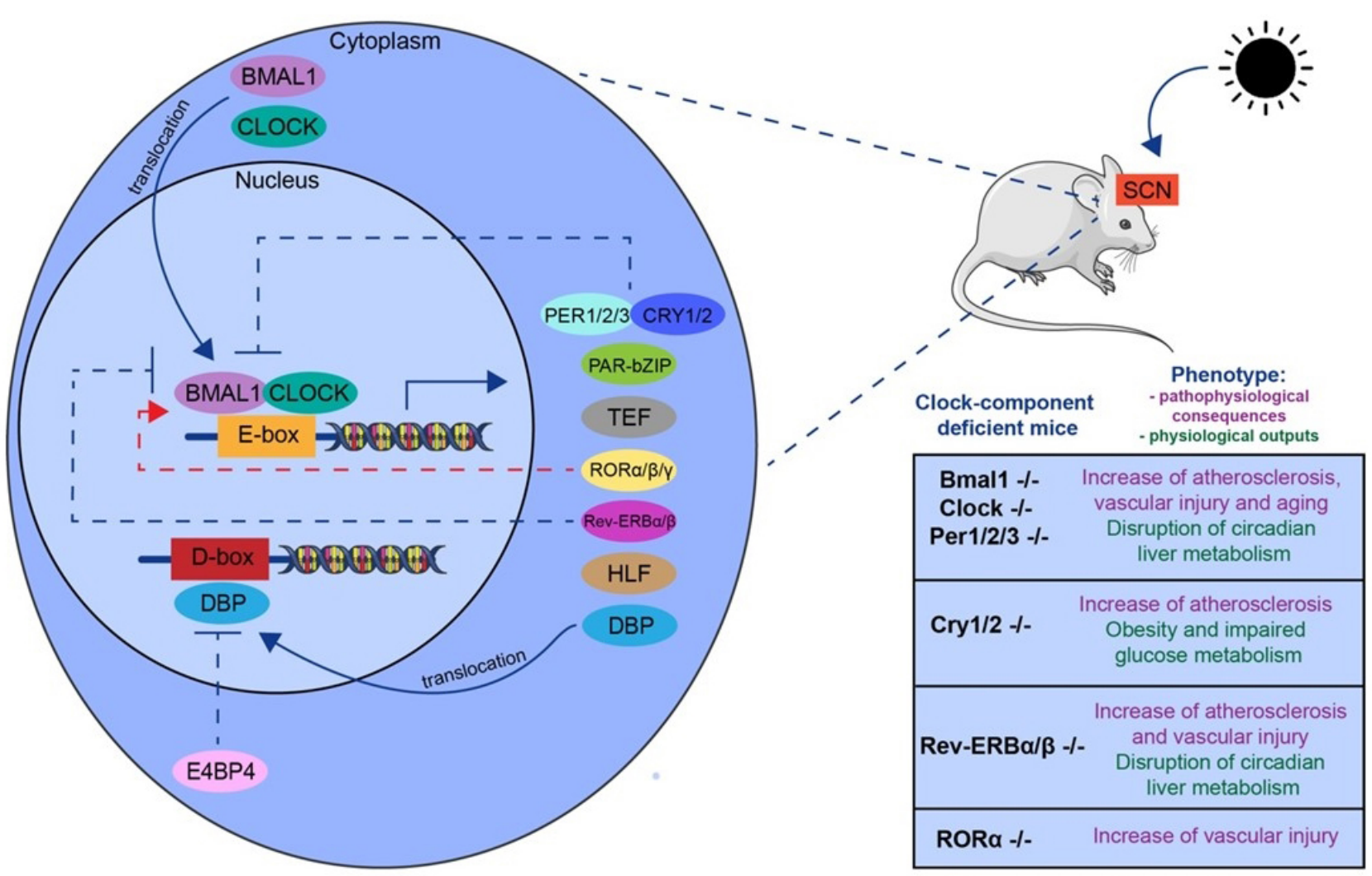

Fig. 2. The mammalian circadian clock in the SCN and its involvement in vascular diseases. CLOCK-BMAL1 heterodimer binding to E-box activates the transcription of different clock genes: PER1/2/3, CRY1/2, Rev-ERVa/b, RORa, HLF, TEF, PIR-bZIP and DBP [33,34,39]. PER and CRY proteins dimerize and block their own transcription by inhibiting CLOCK-BMAL1 activity in the nucleus [36]. Rev-ERB also inhibit CLOCK and BMAL1 expression [2]. This constitutes the negative limb of this transcriptional-translational feedback loop. In the opposite site, ROR proteins positively regulate the expression of CLOCK and BMAL1, constituting the positive limb of the transcriptional-translational feedback loop [2]. Finally, the nuclear factor E4BP4 repress DBP binding to D-box, inhibiting the expression of CCGs, forming the third stabilizing loop [39]. Deficiency or alteration of any of the components of the core circadian clock has an enormous impact at the vascular level, causing atherosclerosis [37-39] and risk of thrombosis [53], alteration of blood hemodynamics [43-47], promoting vascular injury [48], and contributes to aging [49-52]. It also alters liver function [40-42].

Other mechanisms involve hemodynamic and vascular functions. Lack of BMAL1, CLOCK, PER1/2, ROR $\alpha$, or REV-ERB $\alpha / \beta$ in hematopoietic, endothelial, or smooth muscle cells leads to vascular injury, reduced blood flow, and an impaired ability to control blood pressure oscillations $[48-50,63,64]$. Deficiency in BMAL1 or PER2 is characterized by an increased vasoconstrictor response mediated by cyclooxygenase-1 (COX1) and reduced vasorelaxation mediated by decreases in NO and prostaglandin production [51,52]. All these factors increase the risk of hypertension in animal models [53]. The effects of BMAL1 deficiency are cell-type dependent; blood pressure rhythmicity is unaffected by deletion of Bmal1 in mouse cardiomyocytes, but is disrupted by deletion in vascular smooth muscle cells or endothelial cells [65].

The circadian machinery also regulates aging, with deletion of BMAL1 and CLOCK in mice leading to loss of the response to vasopressor stress $[66,67]$. Similarly,
Per1-Per2 mutant mice show accelerated aging in association with altered circadian rhythms [68]. A significant number of CLOCK-regulated genes are associated with the cell cycle and cell proliferation. Wall thickening in CLOCKand PER-deficient mice may be a consequence of collagen accumulation in the medial vessel layer $[66,69]$.

Clock genes and proteins are linked to thrombosis and fibrinolysis factors [70]. For instance, both CLOCK and BMAL regulate PAI-1 gene transcription [71], and deficiency in BMAL1 may be related to high levels of fibrinogen, vWF, and PAI-1, creating a prothrombotic state with a increased risk in thrombosis [72]. The identification of circadian-clock genes that regulate thrombosis could clarify the role of circadian rhythms in the pathophysiology of thrombosis.

Most evidence for the effects of BMAL1-CLOCK, PER1/2/3, and CRY1/2 deficiency comes from studies in mice. Recently, the European Society of Cardiology Work- 
ing Group on Cellular Biology of the Heart published a position paper on the key requirements for preclinical and translational research on circadian rhythms [1], with a view to future trials in humans.

\section{Circadian rhythms and cardiovascular disease}

\subsection{Ischemic heart disease and acute coronary syndrome}

Cardiovascular events occur more frequently in the early morning, with morning rates higher for both AMI and sudden cardiac death. The circadian rhythms of inflammatory cells play a role in AMI, and circulating neutrophils may migrate more avidly to the myocardium in the morning, triggering a stronger proinflammatory response due to increased expression of C-X-C Motif chemokine receptor type 2 (CXCR2) [21]. Some of these findings still need to be validated in humans [1].

In an animal model, morning onset AMI resulted in adverse remodeling with fibrosis and a larger infarct than seen after afternoon onset AMI [73], and morning onset AMI predicted atherosclerosis progression and AMI recurrence [74]. Gene disruption of BMAL-CLOCK (positive circadian component) appears to induce diurnal oscillation of fatty-acid oxidation through the transcriptional activity of the clock protein KLF15 [39]. Specific deletion of BMAL-CLOCK in cardiomyocytes increases infarct size in mice and causes sudden cardiac death, whereas disruption of other clock components, such as PER2 and REV-ERB $\alpha / \beta$ (negative components) reduces infarct size [73,75]. PER2, through the adenosine Adora2b receptor, enhances glycolysis and reduces fatty-acid oxidation in a mechanism dependent on hypoxia-inducible factor (HIF)- $1 \alpha$, leading to reduced infarct size [76].

Similar findings were reported upon deletion of clock genes in fibroblasts, which are involved in post-AMI remodeling. Deletion of fibroblast and cardiomyocyte clock genes led to a decrease in left ventricular ejection fraction and increased left ventricular dimensions [77].

The sleep-wake cycle in mammals is regulated by melatonin, a hormone released by the pineal gland at night. Melatonin has anti-inflammatory and antioxidant properties and this diurnal hormonal regulation is associated with cardiac protection, with melatonin levels correlating with a decreased risk of AMI [78]. Melatonin protects cardiac microvascular endothelial cells by inhibiting autophagy after ischemia-reperfusion injury via the AMP activated protein kinase/mammalian target of rapamycin (AMPK/mTOR) system [79].

\subsection{Cerebrovascular disease and stroke}

The circadian clock also regulates multiple metabolic functions of the central nervous system (CNS), including the brain and cerebrovascular circulation. As seen with ischemic heart disease, the onset of stroke symptoms shows a diurnal variation [31], with ischemic stroke and transient ischemic attack both predominantly occurring between 6 am and noon. Intracerebral hemorrhage (ICH) and subarachnoid hemorrhage onset shows two peaks (albeit less pronounced than ischemic stroke), with a main peak in the morning and a second in the evening [80]. A diurnal variation in the severity of ICH has been reported, with higher 30-day mortality for patients with morning onset [81].

Circadian rhythms in the brain affect molecular mechanisms, mitochondrial function, and adenosine triphosphate (ATP) levels. At the tissue level, the response of the brain to ischemia is dependent on the time of stroke onset. HIF$1-\alpha$ (the primary mediator of hypoxia) interacts with the core circadian genes CLOCK and PER2 [82]. Excitotoxicity and oxidative stress affect neurons, and both processes are influenced by circadian rhythms. In mouse models of brain damage, levels of the NMDA receptor and glutamate (responsible for neurotoxicity) differed depending on what time of day the injury occurred [83]. The diurnal variations in glutamate concentration may be related to daily changes in the endogenous production of melatonin, which has neuroprotective and antioxidant properties [84].

In animal models, astrocytes deficient in BMAL1, CLOCK, or PER2 are more vulnerable to reactive oxygen species (ROS) [85-87]. This effect is thought to involve alterations to regulatory processes such as ATP release from astrocytes and oligodendrocytes, reflecting an influence of circadian signaling on glial cells [88]. Circadian genes also regulate endothelial nitric oxide synthase (eNOS), influencing oscillations in the vascular tone of the cerebral arteries, with important implications for ischemic and hemorrhagic stroke [51]. Bmall deficiency in mice also affects blood-brain barrier function and permeability [89]. In a mouse model of subarachnoid hemorrhage (SAH), PER2 expression was significantly higher in cells isolated from cerebrospinal fluid after ruptured aneurysm than in cells from unruptured aneurysms, reflecting that clock gene expression might regulates, in part, the severity of $\mathrm{SAH}$, this disturbed molecular circadian rhythms may influence the severity of neuronal injury measured by changes in vasoreactivity, neuronal apoptosis, and myeloid-driven neuroinflammation [90]. Circadian rhythms may also affect stroke recovery, since clock genes are essential for neural stem cell differentiation, and disruption of circadian genes in mice leads to altered neurogenesis [91].

\section{Therapeutic implications of circadian rhythms}

One of the simplest ways to apply knowledge of circadian rhythms is to perform surgical and other procedures at specific times during the day cycle, which has the potential to enhance treatment success for diseases such as cancer [92]. Correcting circadian rhythm imbalances may also provide a route to reducing cardiovascular risk. For example, night-time administration of antihypertensive drugs improves overall 24-hour blood pressure profiles more ef- 
fectively than daytime administration [93]. Moreover, lowdose aspirin is more effective at reducing morning platelet reactivity via cyclooxygenase 1 when administered in the evening rather than in the morning [94]; this may reflect circadian differences in the pharmacokinetics and pharmacodynamics or in the specific target response [1].

Chronopharmacology-based approaches could also be used to synchronize the targeting of inflammatory cell populations with their circadian patterns. For example, this strategy could increase the benefit of treatment with the beta-blocker metoprolol, which reduces infarct size after AMI by interfering with neutrophil and platelet interactions and neutrophil recruitment [95]. Similarly, inhibition of CXCR2 in mice represses leukocyte recruitment to plaques when the treatment is administered at night (ZT17), whereas daytime treatment (ZT5) has no therapeutic benefit [96].

Mouse studies have explored the possibility of modulating circadian rhythms by targeting clock genes. One example is the synthetic REV/ERB $\alpha$ ligand GSK4112, which modulates inflammation by attenuating macrophage cytokine production [61]. The REV/ERB agonist SR9009 is able to suppress atherosclerosis in mice [97], and an antiinflammatory effect was detected upon targeting of CRY $1 / 2$ with the activator KL001 in vitro [98].

Another potential therapeutic target is the endovascular protective factor STIR1, a nicotinamide adenine dinucleotide (NAD+)-dependent protein capable of deacetylating transcription factors implicated in inflammatory processes, such as NF/kB [99]. In cell models, reduced levels of STIR1 are associated with altered lipid metabolism and increased foam cell formation [100]. Mice with STIR1 deficiency have altered expression of PER1/2, BMAL1CLOCK, and CRY1/2, suggesting an involvement in circadian rhythms [101]. STIR1 may affect the circadian levels of metabolites involved in plaque formation, such as acetyl-CoA and NAD+ [102]. In general, STIR1 appears to function as an important link between circadian clock genes and lipid metabolism, suggesting that STIR1 activators could be used to prevent atherosclerosis [16]. This potential has been demonstrated for the STIR1 activator resveratrol, a polyphenol (a plant secondary metabolite). Resveratrol has antioxidant properties and has been shown to reduce obesity rates in fat-fed mice by reducing the circadian expression of the clock genes BMAL1, CLOCK, and PER2, as well as clock-controlled genes related to lipid metabolism, such as Sirt1, Srebp-1c, Ppar $\alpha$, Acc1, and Fas [103]. Other polyphenols that interact with circadian genes, such as equol, have been shown to improve lipid profile by increasing HDL-cholesterol and reducing LDL-cholesterol, total cholesterol, and serum triglycerides, resulting in a reduction in plaque formation and atherosclerosis [104].

Krüppel-like factors (KLFs) are transcription factors implicated in cell proliferation and differentiation. When overexpressed specifically in endothelial cells, KLFs can reduce atherosclerosis by interfering with vascular smooth cell differentiation genes [105]. BMAL1-CLOCK can directly regulate the KLF transcription rate, reflecting the importance of circadian rhythms in this process [106]. Besides its anti-inflammatory effects, KLF2 shows antithrombotic activity, increasing the expression of anti-thrombotic molecules such as thrombomodulin-1 and preventing the binding of thrombin to its receptor, protease-activated receptor 1 (PAR-1) [107].

\section{Conclusions}

Emerging evidence from experimental and clinical studies suggests that circadian rhythms driven by the molecular clock play a prominent role in the pathophysiology of thrombosis and atherothrombotic events, influencing or precipitating a state of pathological vascular remodeling, endothelial dysfunction, and thrombosis when dysregulated. Targeting circadian mechanisms and molecules may provide therapeutic benefit by interfering with pathological processes and decreasing cardiovascular morbidity and mortality. Further research is warranted to fully define circadian mechanisms and their relationship with cardiovascular disease.

\section{Abbreviations}

SCN, hypothalamic suprachiasmatic nucleus; AT, antithrombin; PAI-1, plasminogen activator inhibitor-1; BTG, B-thromboglobulin; ON, nitric oxide; AMI, acute myocardial infarction; BMAL1 or ARNTL, brain-muscle aryl hydrocarbon receptor nuclear translocator-like protein 1; CLOCK, circadian locomotor output cycles kaput; ROR, receptor-related orphan receptors; PAR-bZIP, proline and acidic amino acid-rich basic leucine zipper; TEF, thyrotroph embryonic factor; DBP, D-box binding protein; HLF, hepatic leukemia factor; MCP-1, monocyte chemoattractant protein-1; TFN- $\alpha$, tumor necrosis factor- $\alpha$; PPAR, peroxisome proliferating activated receptor; COX1, cyclooxygenase-1; CXCR2, C-X-C motif chemokine receptor type 2; HIF, hypoxia-inducible factor; AMPK/mTOR, AMP activated protein kinase/mammalian target of rapamycin; ICH, intracerebral hemorrhage; ATP, adenosine triphosphate; ROS, reactive oxygen species; eNOS, endothelial nitric oxide synthase; KLF, Krüppel-like factor; PAR-1, protease-activated receptor 1.

\section{Author contributions}

HB and EPM designed the review. EPM performed the research. EPM wrote the manuscript. AAC and $\mathrm{AH}$ provided help and advice on the research topic, figures and references. GM, SH, LV and NR provided help and advice on references and editing. All authors contributed to editorial changes to the manuscript. All authors read and approved the final manuscript. 


\section{Ethics approval and consent to participate}

Not applicable.

\section{Acknowledgment}

We would like to thank the reviewers for their comments and suggestions.

\section{Funding}

This research received no external funding.

\section{Conflict of interest}

$\mathrm{HB}$ receives research funding from the Instituto de Salud Carlos III, Spain (PI17/01799 \& PI21/01572), Sociedad Española de Cardiología, Astra-Zeneca, Bayer, PhaseBio and Novartis and has received consulting fees from Astra-Zeneca and Novartis and speaking fees from Novartis and MEDSCAPE-the heart.og. AH is a paid consultant for Flagship Pioneering. EPM, GM, AAC, SH, LV and NR have no conflicts of interest.

\section{References}

[1] Lecour S, Du Pré BC, Bøtker HE, Brundel BJJM, Daiber A, Davidson SM, et al. Circadian rhythms in ischaemic heart disease. Key aspects for preclinical and translational research: Position paper of the ESC Working Group on Cellular Biology of the Heart. Cardiovascular Research. 2021. (in press)

[2] Reppert SM, Weaver DR. Coordination of circadian timing in mammals. Nature. 2002; 418: 935-941.

[3] Sharma VK. Adaptive significance of circadian clocks. Chronobiology International. 2003; 20: 901-919.

[4] Reppert SM, Weaver DR. Molecular analysis of mammalian circadian rhythms. Annual Review of Physiology. 2001; 63: 647676.

[5] Mohawk JA, Green CB, Takahashi JS. Central and peripheral circadian clocks in mammals. Annual Review of Neuroscience. 2012; 35: 445-462.

[6] Degaute JP, van de Borne P, Linkowski P, Van Cauter E. Quantitative analysis of the 24-hour blood pressure and heart rate patterns in young men. Hypertension. 1991; 18: 199-210.

[7] Nakagawa M, Iwao T, Ishida S, Yonemochi H, Fujino T, Saikawa T, et al. Circadian rhythm of the signal averaged electrocardiogram and its relation to heart rate variability in healthy subjects. Heart. 1998; 79: 493-496.

[8] Dilaveris PE, Färbom P, Batchvarov V, Ghuran A, Malik M. Circadian behavior of P-wave duration, $\mathrm{P}$-wave area, and PR interval in healthy subjects. Annals of Noninvasive Electrocardiology. 2001; 6: 92-97.

[9] Shea SA, Hilton MF, Hu K, Scheer FAJL. Existence of an endogenous circadian blood pressure rhythm in humans that peaks in the evening. Circulation Research. 2011; 108: 980-984.

[10] Oosting J, Struijker-Boudier HA, Janssen BJ. Autonomic control of ultradian and circadian rhythms of blood pressure, heart rate, and baroreflex sensitivity in spontaneously hypertensive rats. Journal of Hypertension. 1997; 15: 401-410.

[11] Idema RN, van den Meiracker AH, Balk AH, Bos E, Schalekamp MA, Man in 't Veld AJ. Abnormal diurnal variation of blood pressure, cardiac output, and vascular resistance in cardiac transplant recipients. Circulation. 1994; 90: 2797-2803.

[12] Kawano H, Motoyama T, Yasue H, Hirai N, Waly HM, Kugiyama K, et al. Endothelial function fluctuates with diurnal variation in the frequency of ischemic episodes in patients with variant angina. Journal of the American College of Cardiology. 2002; 40: 266-270.

[13] Walters J, Skene D, Hampton SM, Ferns GA. Biological rhythms, endothelial health and cardiovascular disease. Medical Science Monitor : International Medical Journal of Experimental and Clinical Research. 2003; 9: RA1-RA8.

[14] Vetter C, Devore EE, Wegrzyn LR, Massa J, Speizer FE, Kawachi I, et al. Association between Rotating Night Shift Work and Risk of Coronary Heart Disease among Women. JAMA. 2016; 315: 1726-1734.

[15] Lusis AJ. Atherosclerosis. Nature. 2000; 407: 233-241.

[16] Man AWC, Li H, Xia N. Circadian Rhythm: Potential Therapeutic Target for Atherosclerosis and Thrombosis. International Jounal of Molecular Sciences. 2021; 22: 676.

[17] Libby P, Ridker PM, Hansson GK. Progress and challenges in translating the biology of atherosclerosis. Nature. 2011; 473: 317-325.

[18] Moore KJ, Sheedy FJ, Fisher EA. Macrophages in atherosclerosis: a dynamic balance. Nature Reviews. Immunology. 2013; 13: 709-721.

[19] Aroca-Crevillén A, Adrover JM, Hidalgo A. Circadian Features of Neutrophil Biology. Frontiers in Immunology. 2020; 11: 576.

[20] Hidalgo A, Chilvers ER, Summers C, Koenderman L. The Neutrophil Life Cycle. Trends in Immunology. 2019; 40: 584-597.

[21] Schloss MJ, Horckmans M, Nitz K, Duchene J, Drechsler M, Bidzhekov $\mathrm{K}$, et al. The time-of-day of myocardial infarction onset affects healing through oscillations in cardiac neutrophil recruitment. EMBO Molecular Medicine. 2016; 8: 937-948.

[22] Rudnicka AR, Rumley A, Lowe GDO, Strachan DP. Diurnal, seasonal, and blood-processing patterns in levels of circulating fibrinogen, fibrin D-dimer, C-reactive protein, tissue plasminogen activator, and von Willebrand factor in a 45 -year-old population. Circulation. 2007; 115: 996-1003.

[23] Scheer FAJL, Shea SA. Human circadian system causes a morning peak in prothrombotic plasminogen activator inhibitor-1 (PAI-1) independent of the sleep/wake cycle. Blood. 2014; 123: 590-593.

[24] Pritchett D, Reddy AB. Circadian Clocks in the Hematologic System. Journal of Biological Rhythms. 2015; 30: 374-388.

[25] Andrews NP, Gralnick HR, Merryman P, Vail M, Quyyumi AA. Mechanisms underlying the morning increase in platelet aggregation: a flow cytometry study. Journal of the American College of Cardiology. 1996; 28: 1789-1795.

[26] Jafri SM, VanRollins M, Ozawa T, Mammen EF, Goldberg AD, Goldstein S. Circadian variation in platelet function in healthy volunteers. The American Journal of Cardiology. 1992; 69: 951-954.

[27] Kanabrocki EL, George M, Hermida RC, Messmore HL, Ryan $\mathrm{MD}$, Ayala DE, et al. Day-night variations in blood levels of nitric oxide, T-TFPI, and E-selectin. Clinical and Applied Thrombosis/Hemostasis. 2001; 7: 339-345.

[28] Verma S, Anderson TJ. Fundamentals of endothelial function for the clinical cardiologist. Circulation. 2002; 105: 546-549.

[29] Thosar SS, Butler MP, Shea SA. Role of the circadian system in cardiovascular disease. The Journal of Clinical Investigation. 2018; 128: 2157-2167.

[30] Cohen MC, Rohtla KM, Lavery CE, Muller JE, Mittleman MA Meta-analysis of the morning excess of acute myocardial infarction and sudden cardiac death. The American Journal of Cardiology. 1997; 79: 1512-1516.

[31] Elliott WJ. Circadian variation in the timing of stroke onset: a meta-analysis. Stroke. 1998; 29: 992-996.

[32] Johnstone MT, Mittleman M, Tofler G, Muller JE. The pathophysiology of the onset of morning cardiovascular events. American Journal of Hypertension. 1996; 9: 22S-28S. 
[33] Muller JE. Circadian variation in cardiovascular events. American Journal of Hypertension. 1999; 12: 35S-42S.

[34] Meléndez-Fernández OH, Walton JC, DeVries AC, Nelson RJ. Clocks, Rhythms, Sex, and Hearts: How Disrupted Circadian Rhythms, Time-of-Day, and Sex Influence Cardiovascular Health. Biomolecules. 2021; 11: 883.

[35] Boivin DB, Boudreau P. Impacts of shift work on sleep and circadian rhythms. Pathologie-Biologie. 2014; 62: 292-301.

[36] Reilly DF, Westgate EJ, FitzGerald GA. Peripheral circadian clocks in the vasculature. Arteriosclerosis, Thrombosis, and Vascular Biology. 2007; 27: 1694-1705.

[37] Lee C, Etchegaray JP, Cagampang FR, Loudon AS, Reppert SM. Posttranslational mechanisms regulate the mammalian circadian clock. Cell. 2001; 107: 855-867.

[38] Busino L, Bassermann F, Maiolica A, Lee C, Nolan PM, Godinho SIH, et al. SCFFbxl3 controls the oscillation of the circadian clock by directing the degradation of cryptochrome proteins. Science. 2007; 316: 900-904.

[39] Li MD, Xin H, Yuan Y, Yang X, Li H, Tian D, et al. Circadian Clock-Controlled Checkpoints in the Pathogenesis of Complex Disease. Frontiers in Genetics. 2021;12: 721231.

[40] Martino T, Arab S, Straume M, Belsham DD, Tata N, Cai F, et al. Day/night rhythms in gene expression of the normal murine heart. Journal of Molecular Medicine. 2004; 82: 256-264.

[41] Narasimamurthy R, Hatori M, Nayak SK, Liu F, Panda S, Verma IM. Circadian clock protein cryptochrome regulates the expression of proinflammatory cytokines. Proceedings of the National Academy of Sciences of the United States of America. 2012; 109: 12662-12667.

[42] Curtis AM, Fagundes CT, Yang G, Palsson-McDermott EM, Wochal P, McGettrick AF, et al. Circadian control of innate immunity in macrophages by miR-155 targeting Bmal1. Proceedings of the National Academy of Sciences of the United States of America. 2015; 112: 7231-7236.

[43] Rudic RD, McNamara P, Curtis A, Boston RC, Panda S, Hogenesch JB, et al. BMAL1 and CLOCK, two essential components of the circadian clock, are involved in glucose homeostasis. PLoS Biology. 2004; 2: e377.

[44] Zhang D, Tong X, Arthurs B, Guha A, Rui L, Kamath A, et al. Liver clock protein BMAL1 promotes de novo lipogenesis through insulin-mTORC2-AKT signaling. The Journal of Biological Chemistry. 2014; 289: 25925-25935.

[45] Grimaldi B, Bellet MM, Katada S, Astarita G, Hirayama J, Amin $\mathrm{RH}$, et al. Per2 controls lipid metabolism by direct regulation of PPAR $\gamma$. Cell Metabolism. 2010; 12: 509-520.

[46] Barclay JL, Shostak A, Leliavski A, Tsang AH, Jöhren O, Müller-Fielitz $\mathrm{H}$, et al. High-fat diet-induced hyperinsulinemia and tissue-specific insulin resistance in Cry-deficient mice. American Journal of Physiology-Endocrinology and Metabolism. 2013; 304: E1053-E1063.

[47] Lamia KA, Papp SJ, Yu RT, Barish GD, Uhlenhaut NH, Jonker $\mathrm{JW}$, et al. Cryptochromes mediate rhythmic repression of the glucocorticoid receptor. Nature. 2011; 480: 552-556.

[48] Bhatwadekar AD, Beli E, Diao Y, Chen J, Luo Q, Alex A, et al. Conditional Deletion of Bmall Accentuates Microvascular and Macrovascular Injury. The American Journal of Pathology. 2017; 187: 1426-1435.

[49] Chang L, Xiong W, Zhao X, Fan Y, Guo Y, Garcia-Barrio M, et al. Bmal1 in Perivascular Adipose Tissue Regulates RestingPhase Blood Pressure through Transcriptional Regulation of Angiotensinogen. Circulation. 2018; 138: 67-79.

[50] Saito T. The vascular clock system generates the intrinsic circadian rhythm of vascular contractility. Journal of Smooth Muscle Research. 2015; 51: 95-106.

[51] Anea CB, Cheng B, Sharma S, Kumar S, Caldwell RW, Yao L, et $a l$. Increased superoxide and endothelial no synthase uncoupling in blood vessels of Bmal1-knockout mice. Circulation Research. 2012; 111: 1157-1165.

[52] Viswambharan H, Carvas JM, Antic V, Marecic A, Jud C, Zaugg $\mathrm{CE}$, et al. Mutation of the circadian clock gene per2 alters vascular endothelial function. Circulation. 2007; 115: 2188-2195.

[53] Murata Y, Ueno T, Tanaka S, Kobayashi H, Okamura M, Hemmi $\mathrm{S}$, et al. Identification of Clock Genes Related to Hypertension in Kidney from Spontaneously Hypertensive Rats. American Journal of Hypertension. 2020; 33: 1136-1145.

[54] Koike N, Yoo S, Huang H, Kumar V, Lee C, Kim T, et al. Transcriptional architecture and chromatin landscape of the core circadian clock in mammals. Science. 2012; 338: 349-354.

[55] Harbour VL, Weigl Y, Robinson B, Amir S. Phase differences in expression of circadian clock genes in the central nucleus of the amygdala, dentate gyrus, and suprachiasmatic nucleus in the rat. PLoS ONE. 2014; 9: e103309.

[56] Ripperger JA, Schibler U. Rhythmic CLOCK-BMAL1 binding to multiple E-box motifs drives circadian Dbp transcription and chromatin transitions. Nature Genetics. 2006; 38: 369-374.

[57] Chen L, Yang G. Recent advances in circadian rhythms in cardiovascular system. Frontiers in Pharmacology. 2015; 6: 71.

[58] Lowrey PL, Takahashi JS. Genetics of circadian rhythms in Mammalian model organisms. Advances in Genetics. 2011; 74: 175-230.

[59] Huo M, Huang Y, Qu D, Zhang H, Wong WT, Chawla A, et al. Myeloid Bmall deletion increases monocyte recruitment and worsens atherosclerosis. FASEB Journal. 2017; 31: 1097-1106.

[60] Nguyen KD, Fentress SJ, Qiu Y, Yun K, Cox JS, Chawla A. Circadian gene Bmal1 regulates diurnal oscillations of Ly6C(hi) inflammatory monocytes. Science. 2013; 341: 1483-1488.

[61] Gibbs JE, Blaikley J, Beesley S, Matthews L, Simpson KD, Boyce $\mathrm{SH}$, et al. The nuclear receptor REV-ERB $\alpha$ mediates circadian regulation of innate immunity through selective regulation of inflammatory cytokines. Proceedings of the National Academy of Sciences of the United States of America. 2012; 109: 582-587.

[62] Everett LJ, Lazar MA. Nuclear receptor Rev-erb $\alpha$ : up, down, and all around. Trends in Endocrinology and Metabolism. 2014; 25: 586-592.

[63] Ma H, Zhong W, Jiang Y, Fontaine C, Li S, Fu J, et al. Increased atherosclerotic lesions in LDL receptor deficient mice with hematopoietic nuclear receptor Rev-erb $\alpha$ knock- down. Journal of the American Heart Association. 2013; 2: e000235.

[64] Nakashima A, Kawamoto T, Noshiro M, Ueno T, Doi S, Honda $\mathrm{K}$, et al. Dec 1 and CLOCK Regulate $\mathrm{Na}+/ \mathrm{K}+$-ATPase $\beta 1$ Subunit Expression and Blood Pressure. Hypertension. 2018; 72: 746-754.

[65] Xie Z, Su W, Liu S, Zhao G, Esser K, Schroder EA, et al. Smooth-muscle BMAL1 participates in blood pressure circadian rhythm regulation. The Journal of Clinical Investigation. 2015; 125: 324-336.

[66] Antoch MP, Gorbacheva VY, Vykhovanets O, Toshkov IA, Kondratov RV, Kondratova AA, et al. Disruption of the circadian clock due to theClockmutation has discrete effects on aging and carcinogenesis. Cell Cycle. 2008; 7: 1197-1204.

[67] Wang C, Wen M, Wang H, Hsieh I, Li Y, Liu P, et al. Increased vascular senescence and impaired endothelial progenitor cell function mediated by mutation of circadian gene per2. Circulation. 2008; 118: 2166-2173.

[68] Lee CC. The circadian clock and tumor suppression by mammalian period genes. Methods in Enzymology. 2005; 393: 852861.

[69] Miller BH, McDearmon EL, Panda S, Hayes KR, Zhang J, Andrews JL, et al. Circadian and CLOCK-controlled regulation of the mouse transcriptome and cell proliferation. Proceedings of the National Academy of Sciences of the United States of Amer- 
ica. 2007 ; 104: 3342-3347.

[70] Tsimakouridze E V, Alibhai FJ, Martino TA. Therapeutic applications of circadian rhythms for the cardiovascular system. Frontiers in Pharmacology. 2015; 6: 77.

[71] Schoenhard JA, Smith LH, Painter CA, Eren M, Johnson $\mathrm{CH}$, Vaughan DE. Regulation of the PAI-1 promoter by circadian clock components: differential activation by BMAL1 and BMAL2. Journal of Molecular and Cellular Cardiology. 2003; 35: 473-481.

[72] Somanath PR, Podrez EA, Chen J, Ma Y, Marchant K, Antoch $\mathrm{M}$, et al. Deficiency in core circadian protein Bmall is associated with a prothrombotic and vascular phenotype. Journal of Cellular Physiology. 2011; 226: 132-140.

[73] Durgan DJ, Pulinilkunnil T, Villegas-Montoya C, Garvey ME, Frangogiannis NG, Michael LH, et al. Short communication: ischemia/reperfusion tolerance is time-of-day-dependent: mediation by the cardiomyocyte circadian clock. Circulation Research. 2010; 106: 546-550.

[74] Nakashima H, Mashimo Y, Kurobe M, Muto S, Furudono S, Maemura K. Impact of Morning Onset on the Incidence of Recurrent Acute Coronary Syndrome and Progression of Coronary Atherosclerosis in Acute Myocardial Infarction. Circulation Journal. 2017; 81: 361-367.

[75] Schroder EA, Burgess DE, Zhang X, Lefta M, Smith JL, Patwardhan A, et al. The cardiomyocyte molecular clock regulates the circadian expression of Kcnh2 and contributes to ventricular repolarization. Heart Rhythm. 2015; 12: 1306-1314.

[76] Eckle T, Hartmann K, Bonney S, Reithel S, Mittelbronn M, Walker LA, et al. Adora2b-elicited per2 stabilization promotes a HIF-dependent metabolic switch crucial for myocardial adaptation to ischemia. Nature Medicine. 2012; 18: 774-782.

[77] Bennardo M, Alibhai F, Tsimakouridze E, Chinnappareddy N, Podobed P, Reitz C, et al. Day-night dependence of gene expression and inflammatory responses in the remodeling murine heart post-myocardial infarction. American Journal of Physiology. Regulatory, Integrative and Comparative Physiology. 2016; 311: R1243-R1254.

[78] Lochner A, Marais E, Huisamen B. Melatonin and cardioprotection against ischaemia/reperfusion injury: what's new? A review. Journal of Pineal Research. 2018; 65: e12490.

[79] Chen W, Liu H, Chen Y, Sha Y, Ma Q, Zhu P, et al. Melatonin Attenuates Myocardial Ischemia/Reperfusion Injury by Inhibiting Autophagy via an AMPK/mTOR Signaling Pathway. Cellular Physiology and Biochemistry. 2018; 47: 2067-2076.

[80] Butt M-U-RA, Zakaria M, Hussain HM. Circadian pattern of onset of ischaemic and haemorrhagic strokes, and their relation to sleep/wake cycle. Journal of Pakistan Medical Association. 2009; 59: 129-132.

[81] Turin TC, Kita Y, Rumana N, Takashima N, Ichikawa M, Sugihara $\mathrm{H}$, et al. Diurnal variation in onset of hemorrhagic stroke is independent of risk factor status: Takashima Stroke Registry. Neuroepidemiology. 2010; 34: 25-33.

[82] Kobayashi M, Morinibu A, Koyasu S, Goto Y, Hiraoka M, Harada H. A circadian clock gene, per2, activates HIF-1 as an effector molecule for recruitment of HIF- $1 \alpha$ to promoter regions of its downstream genes. FEBS Journal. 2017; 284: 3804-3816.

[83] Estrada-Rojo F, Morales-Gomez J, Coballase-Urrutia E, Martinez-Vargas M, Navarro L. Diurnal variation of NMDA receptor expression in the rat cerebral cortex is associated with traumatic brain injury damage. BMC Research Notes. 2018; 11: 150.

[84] Marquez de Prado B, Castañeda TR, Galindo A, del Arco A, Segovia G, Reiter RJ, et al. Melatonin disrupts circadian rhythms of glutamate and GABA in the neostriatum of the aware rat: a microdialysis study. Journal of Pineal Research. 2000; 29: 209-216.
[85] Lananna BV, Nadarajah CJ, Izumo M, Cedeño MR, Xiong DD, Dimitry J, et al. Cell-Autonomous Regulation of Astrocyte Activation by the Circadian Clock Protein BMAL1. Cell Reports. 2018; 25: 1-9.e5.

[86] Brancaccio M, Patton AP, Chesham JE, Maywood ES, Hastings MH. Astrocytes Control Circadian Timekeeping in the Suprachiasmatic Nucleus via Glutamatergic Signaling. Neuron. 2017; 93: 1420-1435.e5.

[87] Magnone MC, Langmesser S, Bezdek AC, Tallone T, Rusconi $\mathrm{S}$, Albrecht U. The Mammalian circadian clock gene per2 modulates cell death in response to oxidative stress. Frontiers in Neurology. 2014; 5: 289.

[88] Womac AD, Burkeen JF, Neuendorff N, Earnest DJ, Zoran MJ. Circadian rhythms of extracellular ATP accumulation in suprachiasmatic nucleus cells and cultured astrocytes. European Journal of Neuroscience. 2009; 30: 869-876.

[89] Nakazato R, Kawabe K, Yamada D, Ikeno S, Mieda M, Shimba $\mathrm{S}$, et al. Disruption of Bmal1 Impairs Blood-Brain Barrier Integrity via Pericyte Dysfunction. The Journal of Neuroscience. 2017; 37: 10052-10062.

[90] Schallner N, Lieberum J, Gallo D, LeBlanc RH, Fuller PM, Hanafy KA, et al. Carbon Monoxide Preserves Circadian Rhythm to Reduce the Severity of Subarachnoid Hemorrhage in Mice. Stroke. 2017; 48: 2565-2573.

[91] Lo EH, Albers GW, Dichgans M, Donnan G, Esposito E, Foster $\mathrm{R}$, et al. Circadian Biology and Stroke. Stroke. 2021; 52: 2180 2190.

[92] Scheiermann C, Gibbs J, Ince L, Loudon A. Clocking in to immunity. Nature Reviews Immunology. 2018; 18: 423-437.

[93] Bowles NP, Thosar SS, Herzig MX, Shea SA. Chronotherapy for Hypertension. Current Hypertension Reports. 2018; 20: 97.

[94] Bonten TN, Snoep JD, Assendelft WJJ, Zwaginga JJ, Eikenboom J, Huisman MV, et al. Time-Dependent Effects of Aspirin on Blood Pressure and Morning Platelet Reactivity. Hypertension. 2015; 65: 743-750.

[95] García-Prieto J, Villena-Gutiérrez R, Gómez M, Bernardo E, Pun-García A, García-Lunar I, et al. Neutrophil stunning by metoprolol reduces infarct size. Nature Communications. 2017; 8: 14780.

[96] Winter C, Silvestre-Roig C, Ortega-Gomez A, Lemnitzer P, Poelman H, Schumski A, et al. Chrono-pharmacological Targeting of the CCL2-CCR2 Axis Ameliorates Atherosclerosis. Cell Metabolism. 2018; 28: 175-182.e5.

[97] Sitaula S, Billon C, Kamenecka TM, Solt LA, Burris TP. Suppression of atherosclerosis by synthetic REV-ERB agonist. Biochemical and Biophysical Research Communications. 2015; 460: $566-571$.

[98] Hand LE, Hopwood TW, Dickson SH, Walker AL, Loudon ASI, Ray DW, et al. The circadian clock regulates inflammatory arthritis. FASEB Journal. 2016; 30: 3759-3770.

[99] Yeung F, Hoberg JE, Ramsey CS, Keller MD, Jones DR, Frye $\mathrm{RA}$, et al. Modulation of NF-kappaB-dependent transcription and cell survival by the SIRT1 deacetylase. EMBO Journal. 2004; 23: 2369-2380.

[100] Zeng H, Fu Y, Yu W, Lin J, Liang-Zhou, Liu L, et al. SIRT1 prevents atherosclerosis via liver-X-receptor and NF- $\mathrm{kB}$ signaling in a U937 cell model. Molecular Medicine Reports. 2013; 8: 23-28.

[101] Wang R, Zhao T, Cui K, Hu G, Chen Q, Chen W, et al. Negative reciprocal regulation between Sirt1 and per2 modulates the circadian clock and aging. Scientific Reports. 2016; 6: 28633.

[102] Zhu Z, Hua B, Shang Z, Yuan G, Xu L, Li E, et al. Altered Clock and Lipid Metabolism-Related Genes in Atherosclerotic Mice Kept with Abnormal Lighting Condition. BioMed Research International. 2016; 2016: 1-14.

[103] Sun L, Wang Y, Song Y, Cheng X, Xia S, Rahman MRT, et 
al. Resveratrol restores the circadian rhythmic disorder of lipid metabolism induced by high-fat diet in mice. Biochemical and Biophysical Research Communications. 2015; 458: 86-91.

[104] Zhang T, Hu Q, Shi L, Qin L, Zhang Q, Mi M. Equol Attenuates Atherosclerosis in Apolipoprotein E-Deficient Mice by Inhibiting Endoplasmic Reticulum Stress via Activation of Nrf2 in Endothelial Cells. PLoS ONE. 2016; 11: e0167020.

[105] Hergenreider E, Heydt S, Tréguer K, Boettger T, Horrevoets AJG, Zeiher AM, et al. Atheroprotective communication between endothelial cells and smooth muscle cells through miR-
NAs. Nature Cell Biology. 2012; 14: 249-256.

[106] Yoshitane H, Ozaki H, Terajima H, Du N, Suzuki Y, Fujimori $\mathrm{T}$, et al. CLOCK-controlled polyphonic regulation of circadian rhythms through canonical and noncanonical E-boxes. Molecular and Cellular Biology. 2014; 34: 1776-1787.

[107] Pathak R, Shao L, Chafekar SM, Feng W, Ponnappan U, Fink $\mathrm{LM}$, et al. IKK $\beta$ regulates endothelial thrombomodulin in a Klf2-dependent manner. Journal of Thrombosis Haemostasis. 2014; 12: 1533-1544. 